\title{
Regional cerebral blood flow as predictor of response to occipital nerve block in cluster headache
}

\author{
Sonia Medina ${ }^{1,2^{*}+}$ (D) Norazah Abu Bakar ${ }^{3 \dagger}$, Owen O'Daly ${ }^{1}$, Sarah Miller ${ }^{4}$, Elena Makovac ${ }^{1,2}$, Tara Renton ${ }^{3}$,
} Steve C. R. Williams ${ }^{1}$, Manjit Matharu ${ }^{4+}$ and Matthew A. Howard ${ }^{1+}$

\begin{abstract}
Background: Cluster headache is an excruciating disorder with no cure. Greater occipital nerve blockades can transiently suppress attacks in approximately $50 \%$ of patients, however, its mechanism of action remains uncertain, and there are no reliable predictors of treatment response. To address this, we investigated the effect of occipital nerve blockade on regional cerebral blood flow ( $\mathrm{rCBF}$ ), an index of brain activity, and differences between treatment responders and non-responders. Finally, we compared baseline perfusion maps from patients to a matched group of healthy controls.
\end{abstract}

Methods: 21 male, treatment-naive patients were recruited while in a cluster headache bout. During a pain-free phase between headaches, patients underwent pseudo-continuous arterial spin labelled MRI assessments to provide quantitative indices of rCBF. MRIs were performed prior to and 7-to-21 days following treatment. Patients also recorded the frequency of their headache attacks in a daily paper diary. Neuropsychological assessment including anxiety, depression and quality of life measures was performed in a first, scanning free session for each patient.

Results: Following treatment, patients demonstrated relative rCBF reductions in posterior temporal gyrus, cerebellum and caudate, and rCBF increases in occipital cortex. Responders demonstrated relative rCBF increases, compared to non-responders, in medial prefrontal cortex and lateral occipital cortex at baseline, but relative reductions in cingulate and middle temporal cortices. rCBF was increased in patients compared to healthy controls in cerebellum and hippocampus, but reduced in orbitofrontal cortex, insula and middle temporal gyrus.

Conclusions: We provide new mechanistic insights regarding the aetiology of cluster headache, the mechanisms of action of occipital nerve blockades and potential predictors of treatment response. Future investigation should determine whether observed effects are reproducible and extend to other headache disorders.

\footnotetext{
* Correspondence: Sonia.medina@kcl.ac.uk

†Sonia Medina, Norazah Abu Bakar, Manjit Matharu and Matthew A. Howard contributed equally to this work.

'Department of Neuroimaging, King's College London, Institute of Psychiatry, Psychology and Neuroscience, Box 89, De Crespigny Park, London SE5 8AF, UK

${ }^{2}$ Wolfson Centre for Age-Related Diseases, King's College London, London,

UK

Full list of author information is available at the end of the article
}

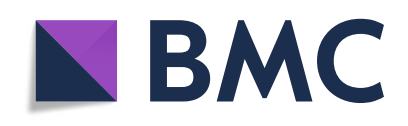

(- The Author(s). 2021 Open Access This article is licensed under a Creative Commons Attribution 4.0 International License, which permits use, sharing, adaptation, distribution and reproduction in any medium or format, as long as you give appropriate credit to the original author(s) and the source, provide a link to the Creative Commons licence, and indicate if changes were made. The images or other third party material in this article are included in the article's Creative Commons licence, unless indicated otherwise in a credit line to the material. If material is not included in the article's Creative Commons licence and your intended use is not permitted by statutory regulation or exceeds the permitted use, you will need to obtain permission directly from the copyright holder. To view a copy of this licence, visit http://creativecommons.org/licenses/by/4.0/. The Creative Commons Public Domain Dedication waiver (http://creativecommons.org/publicdomain/zero/1.0/) applies to the data made available in this article, unless otherwise stated in a credit line to the data. 
Keywords: Cluster headache, Greater occipital nerve block, Regional cerebral blood flow, Arterial spin Labelling,

Trigeminal cephalgia

\section{Introduction}

Cluster headache $(\mathrm{CH})$, a member of the group of trigeminal autonomic cephalgias, is an excruciating condition. It is characterised by strictly unilateral orbital, supraorbital or temporal headaches that severely compromise the quality of life of those who suffer from it. While there are a number of treatments available to alleviate $\mathrm{CH}$ symptoms, at least partially [1], further development is still needed to achieve complete suppression of headache attacks and effective management of commonly associated psychological symptoms (e.g. anxiety, depression).

It is still unclear how some of these therapies work in $\mathrm{CH}$ treatment responders, which suggests the involvement of several interrelated neural processes which require better characterisation. Greater occipital nerve blockade (GONB) is a relatively successful therapy for suppressing $\mathrm{CH}$ attacks with minimal side effects [2]. GONB action is theorised to reduce afferent signalling from the occipital nerve to the sensory trigeminal fibres at the level of the nucleus caudalis, however, the degree of such inhibition is not directly reflected in a proportional reduction of $\mathrm{CH}$ symptoms [3]. A well-defined model that explains how a GONB stops headache attacks, and why it is effective in only a portion of patients who receive it, is yet to be proposed.

Neuroimaging has significantly facilitated our understanding of putative brain mechanisms underpinning $\mathrm{CH}$ [4-7]. Functional magnetic resonance imaging (fMRI) and in particular blood-oxygen-level dependent (BOLD) fMRI, can describe differences in activity and connectivity between $\mathrm{CH}$ patients and healthy controls [8], both in the resting state and during headache attacks $[9,10]$, pointing towards the hypothalamus as a key area involved in triggering headache attacks during bouts, as well as in marking the beginning and end of bouts in episodic $\mathrm{CH}$ patients, causing the circadian nature of $\mathrm{CH}$ symptoms. Nevertheless, these findings continue to be debated, as it remains unclear whether results incorporate the hypothalamus and/or the neighbouring ventral tegmental area (VTA) as the areas responsible for those differences [11]. These contentions are compounded by the small size of these structures and the limited spatial resolution of fMRI. In fact, chronic pain largely relates to spontaneous, low frequency fluctuations, for which arterial spin labelling (ASL) is more optimally sensitive, as it can identify changes in low frequency brain activity via quantification of regional cerebral blood flow (rCBF) as a proxy of resting brain activity in relation to chronic pain [12]. Evidence of $\mathrm{rCBF}$ changes in $\mathrm{CH}$ patients after GONB should therefore provide important new mechanistic insights.

Previous studies have reported decreased metabolism [13] and grey matter volume (GMV) $[14,15]$ in the prefrontal cortex (PFC) in $\mathrm{CH}$ patients in comparison to healthy controls, as well as negative correlations between PFC GMV and disease duration [16]. GMV in medial PFC has been considered as predictor of response to treatments for depression [17] and anxiety disorders [18], both common comorbidities in $\mathrm{CH}$. Accordingly, we hypothesised that prefrontal $\mathrm{rCBF}$ at baseline could relate to the capacity of treatment response, ultimately contributing to differential responses to GONB; therefore we anticipated that prefrontal $\mathrm{CBF}$ at baseline would differ between $\mathrm{CH}$ patients and healthy controls, as well as between those who respond positively to GONB (i.e. responders) and treatment non-responders.

Here, we explored i) rCBF changes in $\mathrm{CH}$ patients following their first GONB treatment to further understand the mechanisms of action of GONB, ii) differences in $\mathrm{rCBF}$ across $\mathrm{CH}$ patients at baseline during interictal phase in relation to response to GONB treatment, and iii) brain perfusion differences between $\mathrm{CH}$ patients at baseline and healthy controls. We hypothesised that a) GONB would result in rCBF differences throughout the brain, b) patterns of baseline rCBF would be useful predictors of treatment response, particularly in the PFC, and c) rCBF would differ between patient and control groups.

\section{Materials and methods \\ Eligibility, groups and screening}

Twenty-one $\mathrm{CH}$ patients (age range: 20-55 years, mean $=37.5 \pm \mathrm{SD}=8.9$ ) were recruited at The National Hospital for Neurology and Neurosurgery in London. Inclusion criteria were: (i) being a male participant; females were excluded from the study to avoid confounds relating to fluctuations in female hormonal levels within and between sessions [19]; (ii) patients diagnosed with $\mathrm{CH}$ according to diagnostic criteria in effect at the time of the study [20] and receiving their first GONB as part of their medical plan; (iii) age range $18-65$ years; (iv) in case of being on preventive medication treatment, a stable dose for a minimum of 1 month [21]; no history or evidence of psychosis, psychological disease, use of recreational drugs or excessive caffeine consumption (i.e. 
more than six cups of caffeinated drinks per day) [21]; no existing major medical problems aside from $\mathrm{CH}$ (e.g. heart disease) and (vii) normal criteria for MRI scanning. Having an abortive treatment within the last $12 \mathrm{~h}$ prior to the scanning sessions was also an exclusion criterion, apart from oxygen treatment. Although existing evidence suggests that brain perfusion likely returns to baseline only a few minutes after a state of hyperoxia, especially under higher concentrations of oxygen [22, 23], oxygen treatment was allowed up to $1 \mathrm{~h}$ before each scanning session to avoid any confounding effects.

In addition, data from seven male, age-matched, physically and psychologically healthy controls from previous studies were included in the last data analysis set. All healthy controls had provided prior written consent for their MRI data to be used in later studies. Exclusion criteria for the recruitment of healthy volunteers included history of brain injuries, hypertension, any psychiatric or neurologic disease, alcohol or drug abuse, insomnia, obstructive sleep apnea, narcolepsy, or restless legs syndrome. Any volunteers that were acutely ill, with fever and malaise, were excluded or rescheduled for examination following complete recovery.

\section{Study design}

A prospective, open-label study was carried out. For all patients, the study required three visits to the imaging centre; (i) a neuropsychological screening and a mock scanning session to familiarise patients to the scanner environment; (ii) a baseline MRI scanning session (including structural T2-weighted images and pCASL measurements) followed by GONB treatment; (iii) a third session, taking place between 7 and 21 days following treatment to examine treatment effects once the effects of the injection were allowed to emerge. At this final session, an MRI scanning session identical to the first was performed (Fig. 1). In addition, for the entire duration of the study, patients were requested to record, on a daily basis, the number of $\mathrm{CH}$ attacks experienced during that day, as well as their duration and severity. All visits were scheduled during patients' bouts in the case of episodic $\mathrm{CH}(\mathrm{ECH})$ patients and between $\mathrm{CH}$ attacks (i.e. pain-free). The average duration of $\mathrm{ECH}$ bouts was taken into account to make sure the last session did not occur during a natural remission of $\mathrm{CH}$ attacks towards the end of a bout, as this could confound the results regarding GONB response. $\mathrm{Pa}$ tients who experience a definite response to GONB after a week were called in earlier to complete their follow up session; on the contrary, patients showing partial/no response to GONB a week after treatment were called in later to ensure enough time was provided for the effects of GONB to be seen and they were not wrongly labelled as non-responders. During MRI scanning, patients and healthy controls were instructed to remain awake in order to control for their level of alertness.

\section{Study procedures}

Neuropsychological assessments were carried out at The National Hospital for Neurology and Neurosurgery in London. All the scanning sessions and GONB were performed at the Centre for Neuroimaging Sciences, Institute of Psychiatry, Psychology and Neuroscience, King's College London.

\section{Neuropsychological assessment}

All $\mathrm{CH}$ patients underwent a neuropsychological assessment on the first visit, including the Hospital Anxiety and Depression Scale (HADS) [24] and MOS 36-Item Short-Form Health Survey (SF-36) to assess patients' quality of life [25].

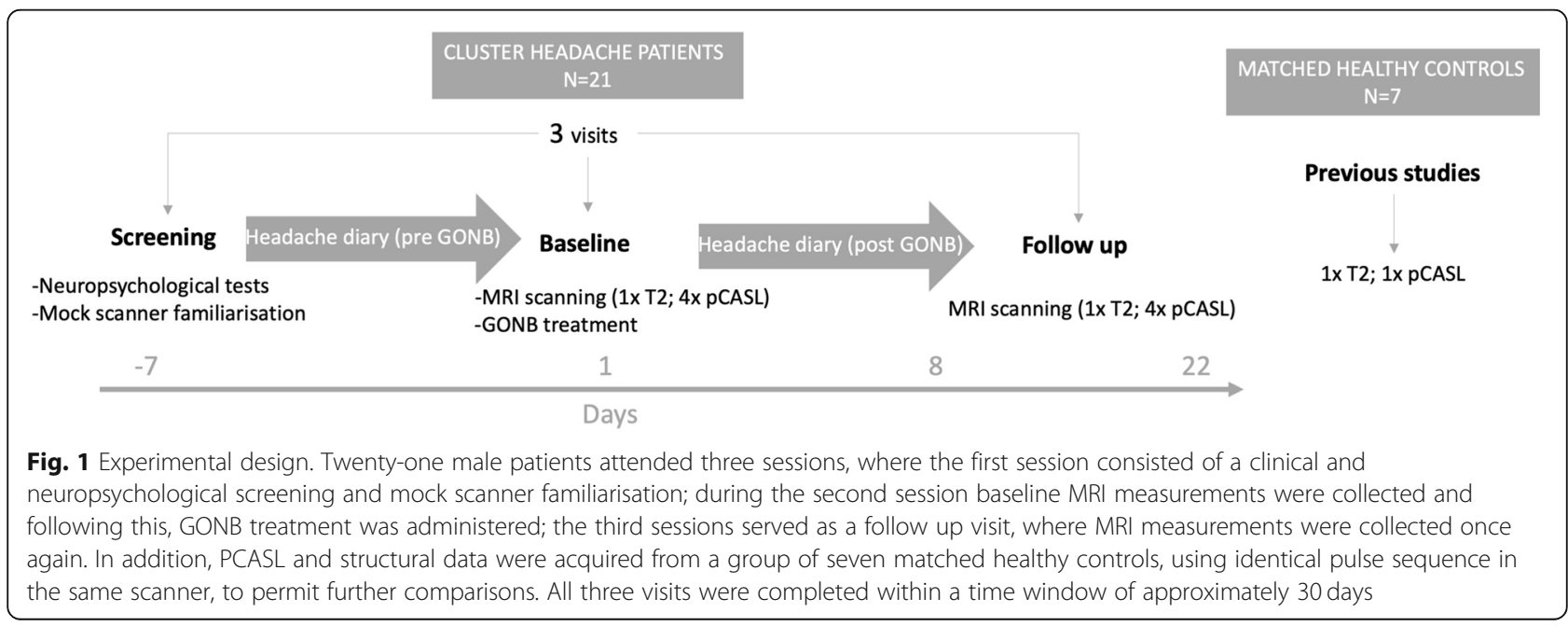




\section{GONB treatment}

The greater occipital nerve block procedure comprised $80 \mathrm{mg}$ methylprednisolone and $2 \mathrm{ml}$ of $2 \%$ lidocaine, injected in the suboccipital area at a point lying on the medial third of a line drawn between the inion and mastoid process ipsilateral to the pain.

\section{MRI acquisition and pre-processing}

MRI imaging was acquired on a $3 \mathrm{~T}$ General Electric Signa HDX whole-body MRI scanner, equipped with an 8-channel, receive-only, phased-array head coil. All patients and healthy controls had an axial $\mathrm{T}_{2}$-weighted $3 \mathrm{D}$ fast-recovered fast spin-echo (FSE) pulse sequence with slice thickness $=2 \mathrm{~mm}$, repetition time $=4380 \mathrm{~ms}$, echo time $=55.44 \mathrm{~ms}$, flip angle $=90^{\circ}$, field of view $=240 \mathrm{~mm}^{2}$, and matrix size $=320 \times 320$. For brain perfusion measurement, a pCASL sequence was used (8 shots, 512 points per spiral arm, repetition time $=1635 \mathrm{~ms}$, echo time $=5.222 \mathrm{~ms}$, pulse duration $=500 \mu \mathrm{s}$, pulse gap $=$ $1500 \mu \mathrm{s}$, post-labelling delay $=1.5 \mathrm{~s}$, voxel size $=1.875 \times$ $\left.1.875 \times 3 \mathrm{~mm}^{3}\right)$. For all $\mathrm{CH}$ patients, regional cerebral blood flow (rCBF) quantification was repeated four times each session. Further details on rCBF computation can be found at https://www.kcl.ac.uk/ioppn/depts/ neuroimaging/research/pain/pCASLdetail.pdf. All MRI measurements were carried out during patients' painfree interictal phases.

Image pre-processing was performed using the FMRIB Software Library (FSL). The first step was skull stripping and segmentation of structural images using FSL BET and FIRST, respectively. T2 scans were normalised to a Montreal Neurological Institute (MNI) T2 template using linear and non-linear registration tools FLIRT and FNIRT respectively. We performed co-registration of pCASL images to native-space T2 skull-stripped scans; the resulting transformation matrix was then inverted and used to co-register normalised T2 scans to pCASL images. Stripped, co-registered pCASL scans were warped into MNI space using linear and non-linear transformation parameters derived resulted from the high-resolution T2-weighted structural images. Images were finally spatially smoothed with an $8 \mathrm{~mm}$ FWHM isotropic Gaussian kernel using Statistical Parametric Mapping software (SPM) version 12 . Quality assurance was manually performed to identify artefacts (e.g. coregistration failures) at each pre-processing step.

\section{Statistical analyses Behavioural data}

The mean and standard deviation of the number of weekly $\mathrm{CH}$ attacks before and after GONB (according to their Headache Diary notes) were computed for each patient. Patients were then divided in two groups: the responders group included patients who experienced a
$50 \%$ or greater reduction in the average of weekly $\mathrm{CH}$ attacks after GONB treatment; the remaining patients were included in the non-responders group. Results from HADS and SF-36 subscales were compared between the responders and non-responders group by means of an independent samples t-test.

\section{Neuroimaging data}

Group wise statistical analyses of pre-processed pCASL images were carried out in SPM 12 using a mass univariate general linear model approach. For inference, an initial uncorrected cluster-forming height threshold was set to $p<0.001$. Results were Family-Wise Error (FWE) corrected on the basis of cluster extent at $p<0.05$ according to Random Field Theory. An explicit grey matter mask template was included in all the designs. Strictly for exploratory purposes, whole brain changes across patients after treatment are also displayed at a less stringent uncorrected cluster-forming height threshold $=0.005$. Effect size statistics (Cohen's d) were computed as a function of the $t$ value for each contrast and the sample size, accounting for both paired [26] or independent samples [27] model designs. In order to avoid inflated effect sizes, that may be biased due to small sample sizes (i.e. $n<20$ ), Hedge's g statistic for corrected effect size were also calculated as a function of Cohen's d results [28].

\section{Changes in rCBF after treatment across patients}

In order to examine regional differences in $\mathrm{CBF}$ before and after treatment, we performed a repeated-measures analysis of variance (ANOVA) with three factors: Subjects, Treatment (pre/post treatment) and Scan (with four levels, one per CBF map). Despite the fact that all patients were scanned during an interictal phase in a pain-free state, $\mathrm{CBF}$ maps from episodic $\mathrm{CH}$ and chronic $\mathrm{CH}$ patients were compared at baseline via an independent sample t-test, to assess the appropriateness of their inclusion in subsequent modelling as a single sample. No significant differences were identified. As $\mathrm{CH}$ attacks are presented unilaterally, we investigated CBF maps from patients reporting attacks on their right side, compared to those reporting attacks on their left, using an independent sample t-test, in order to rule out confounding effects of the headache side. Since no significant differences were observed, in subsequent models information about the laterality of attacks was included in the design as a nuisance covariate. Patients age, duration of $\mathrm{CH}$ (measured in number of years from the first $\mathrm{CH}$ attack to the moment of first visit) and global $\mathrm{CBF}$ signal were also included as additional nuisance covariates. We examined the main effect of Treatment via pretreatment $</>$ post-treatment contrasts. 


\section{Responders vs non responders to GONB treatment}

Scans acquired before treatment for the responders and non-responders group were compared via a two-way analysis of variance (ANOVA) with two factors: Treatment response (responders/non responders) and Scan (four levels, one per $\mathrm{CBF}$ map) to determine whether baseline rCBF could predict response or non-response to GONB treatment in $\mathrm{CH}$. Global CBF, age, duration of $\mathrm{CH}$, global white matter volume and global cerebrospinal fluid volume (CSF) (both measured in millilitres) were included in the analysis as additional nuisance covariates. Two analyses were performed with patients' pre and post treatment images respectively.

In order to test our a priori hypothesis regarding prefrontal local CBF increases in the responders group compared to non-responders, we performed a small volume correction (SVC) for the contrast 'responders > nonresponders'. The frontal cortex region of interest (ROI) was chosen from a predefined mask in SPM12.

\section{$\mathrm{CH}$ patients vs healthy controls}

Baseline pCASL data from all patients were compared with data from an available database of age matched healthy controls, to determine rCBF differences that relate to $\mathrm{CH}$. In this case, only the first $\mathrm{CBF}$ map for each $\mathrm{CH}$ individual was used for analysis, due to data availability limitations from healthy controls. Global CBF, global white matter volume, global CSF volume, and number of months passed from the scanner acquisition date to the analysis date were added to the model as nuisance covariates.

\section{Results}

Two patients failed to complete the last session, and one was excluded due to unrecoverable motion artefacts in MRI data. Data from a total of 18 patients were included in the data analysis.

\section{Behavioural data \\ Headache diary data}

One patient did not provide information for the Headache Diary; results from a total of 17 patients were included in the behavioural data analysis (Tables 1 and 2). Overall, $52 \%$ of patients $(n=9)$ were considered responders to treatment, experiencing a reduction in weekly $\mathrm{CH}$ attacks greater than $50 \%$, six of whom became completely pain free. Eight patients were designated as non-responders; within this group, two patients reported a reduction in weekly attacks lower than 50\%, no changes were reported by two patients and remaining subjects reported an increased number of attacks after treatment. Response to GONB could be determined 1 week after GONB in most cases, except for one patient who showed a positive response in terms of reduction of average weekly attacks after 3 weeks.

\section{HADS and SF-36: responders vs non-responders at baseline}

Due to missing data in questionnaires from two patients, data from a total of 16 participants, eight responders and eight non-responders were included in this analysis (for a summary of results, see Table 2B). Anxiety and depression measures reported by patients were on average between 8 and 10, an interval that corresponds to the category 'borderline abnormal' as specified by HADS score interpretation standards. Quality of life (QoL) scores represent the percentage of total possible scored achieved. We found the lowest scores (below percentile 40) in QoL subscales 'role limitations due to physical health', 'energy/fatigue', 'role limitations due to emotional problems' and 'pain'. Independent samples t-test revealed no significant difference between responders $(n=8)$ and non-responders $(\mathrm{n}=8)$ for all HADS and SF-36 subscales (all $p$ 's $>0.05$, Table 2B). Thus, independently of treatment outcome, responders and non-responders did not differ in depression and anxiety symptoms or quality of life measures at baseline.

\section{Imaging data \\ Regional CBF changes across patients: perfusion before vs after treatment}

We sought to determine differences in rCBF between scans before and after GONB treatment for each patient. Repeated-measures ANOVA indicated a main effect of Treatment (i.e. pre vs post GONB); patients presented local decreases in rCBF after treatment across three main clusters in the left hemisphere, including posterior temporal gyrus, cerebellum and caudate, in comparison to the post GONB session. In contrast, increases in $\mathrm{rCBF}$ after treatment across patients were identified in the right secondary visual cortex (see Fig. 2 and Table 3). Exploratory results at a more liberal cluster-forming threshold $(p<0.005)$ revealed additional decreases in rCBF after treatment in thalamus bilaterally, right hypothalamus and ventral tegmental area (VTA), pons, substantia nigra bilaterally and cerebellum (Fig. 5).

\section{Predicting treatment response: regional CBF differences between responders and non-responders at baseline}

Contrasts comparing CBF maps at baseline (i.e. before GONB treatment) in responders versus non-responders group showed that patients who responded to treatment had greater $\mathrm{rCBF}$ in the right lateral occipital cortex, and lower $\mathrm{rCBF}$ in right posterior cingulate gyrus.

(Fig. 3, Table 3). Our hypothesis-led analysis (i.e. SVC) to test for prefrontal cortical CBF differences between 
Table 1 Demographic and main clinical data from $\mathrm{CH}$ patients included in behavioural data analyses $(N=17)$

\begin{tabular}{|c|c|c|c|c|c|c|}
\hline Patient & Age & Duration $\mathrm{CH}$ & CH side & Type of $\mathrm{CH}$ & Preventive medication & Acute medication \\
\hline \multirow[t]{2}{*}{1} & 39 & 14 & Right & Episodic & - & Triptan \\
\hline & & & & & & Oxygen \\
\hline 2 & 29 & 7 & Right & Episodic & Verapamil & Triptan \\
\hline \multirow[t]{2}{*}{$3^{\mathrm{a}}$} & 46 & 5 & Right & Episodic & - & Triptan \\
\hline & & & & & & Oxygen \\
\hline 4 & 34 & 17 & Left & Episodic & & $\begin{array}{l}\text { Triptan } \\
\text { Oxygen }\end{array}$ \\
\hline \multirow[t]{3}{*}{5} & 50 & 10 & Right & Chronic & Verapamil & Triptan \\
\hline & & & & & Topiramate & Oxygen \\
\hline & & & & & Tricyclic antidepressants & \\
\hline \multirow[t]{2}{*}{6} & 64 & 42 & Right & Episodic & - & Triptan \\
\hline & & & & & & Oxygen \\
\hline 7 & 36 & 7 & Right & Chronic & - & - \\
\hline \multirow[t]{2}{*}{8} & 35 & 7 & Right & Episodic & - & Triptan \\
\hline & & & & & & Oxygen \\
\hline \multirow[t]{2}{*}{9} & 32 & 10 & Left & Chronic & - & Triptan \\
\hline & & & & & & Oxygen \\
\hline 10 & 34 & 11 & Right & Episodic & - & Oxygen \\
\hline \multirow[t]{2}{*}{11} & 34 & 6 & Right & Chronic & Verapamil & Triptan \\
\hline & & & & & & Oxygen \\
\hline \multirow[t]{2}{*}{12} & 43 & 26 & Right & Episodic & - & Triptan \\
\hline & & & & & & Oxygen \\
\hline 13 & 55 & 25 & Right & Episodic & Melatonin & Oxygen \\
\hline \multirow[t]{2}{*}{14} & 20 & 1 & Left & Chronic & Verapamil & Triptan \\
\hline & & & & & & Oxygen \\
\hline 15 & 28 & 13 & Left & Chronic & Verapamil & Oxygen \\
\hline \multirow[t]{3}{*}{16} & 38 & 9 & Left & Episodic & Verapamil & Triptan \\
\hline & & & & & Prednisolone & Oxygen \\
\hline & & & & & Tricyclic antidepressants & \\
\hline \multirow[t]{3}{*}{17} & 47 & 16 & Left & Episodic & Verapamil & Triptan \\
\hline & & & & & Lithium & Oxygen \\
\hline & & & & & Tricyclic antidepressants & \\
\hline $18^{\mathrm{b}}$ & 36 & 7 & Right & Chronic & - & - \\
\hline
\end{tabular}

All patients on preventive medication were on a stable treatment for at least 1 month prior to the first visit and throughout the duration of the study. Participants were instructed to abstain from all medication, apart from oxygen treatment, for at least $12 \mathrm{~h}$ prior to each scanning session. Duration $\mathrm{CH}=$ years passed since diagnosis of $\mathrm{CH}$ to beginning of the study; $\mathrm{CH}$ side = laterality of $\mathrm{CH}$ attacks; $\mathrm{CH}=\mathrm{Cluster}$ Headache

aparticipant excluded from behavioural analyses due to missing data

barticipant excluded from behavioural analyses and treatment response analyses due to missing data

responders and non-responders indicated that patients who responded to GONB treatment had greater $\mathrm{rCBF}$ at baseline in left medial PFC (mPFC), compared to patients who did not experience a substantial improvement after treatment $(p \mathrm{FWE}=0.015, \quad \mathrm{t}$-score $=5.56, \quad 115$ voxels).

\section{Regional CBF differences between responders and non- responders after GONB treatment}

With the purpose of acquiring a better understanding of differences between responders and non-responders, we compared $\mathrm{CBF}$ for both groups across the whole brain after treatment. Following GONB, patients who 
Table 2 Behavioural data from Headache Diary (A), as well as from HADS and SF-36 questionnaires [29]

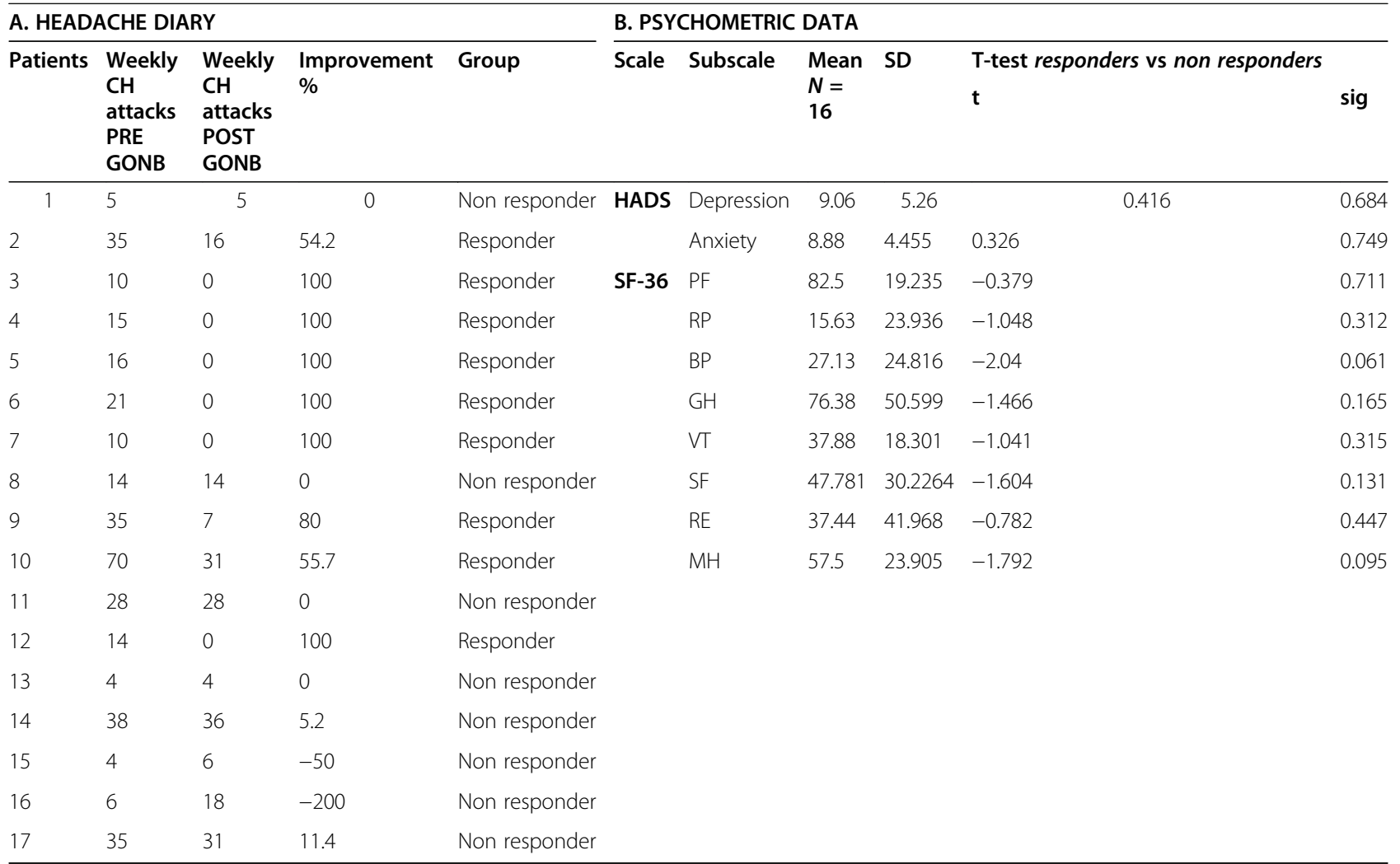

Results from Headache Diary included 17 patients as one of the patients included in the MRI data analysis failed to provide results. Similarly, two patients failed to provide HADS and SF-36 responses, and therefore 16 patients were included in the independent samples t-test (i.e. eight responders and 8 non-responders). $\mathrm{CH}=$ Cluster Headache; GONB = Greater Occipital Nerve Block; HADS = Hospital Anxiety and Depression Scale; SF-36 = MOS 36-Item Short-Form Health

Survey. Alpha $=0.05$

responded to GONB showed greater $\mathrm{rCBF}$ in right lateral occipital cortex, as well as lower rCBF in right posterior cingulate cortex and left middle temporal gyrus than patients who did not respond. SVC in the PFC did not show any significant differences between both groups following treatment.

\section{Regional CBF differences between $\mathrm{CH}$ patients and healthy controls}

We compared pre-treatment rCBF maps with those from a subgroup of healthy individuals (Fig. 4). Relative increases in rCBF in patients, compared to healthy control participants were observed in lobule VIII of left cerebellum and left hippocampus. Comparative reductions in the patient group were identified in the right orbitofrontal cortex, rostral anterior insula and middle temporal gyrus (Table 3).

\section{Discussion}

We examined rCBF changes following GONB in $\mathrm{CH}$ patients, using pCASL fMRI imaging. We explored differences in rCBF between patients who responded to treatment and patients who did not respond, obtaining, to the best of our knowledge, the first evidence of $\mathrm{rCBF}$ differences that may act as predictors of GONB treatment efficacy in $\mathrm{CH}$. Finally, we compared patients' rCBF maps at baseline with matched healthy controls, in order to provide further meaningful information on the pathophysiology of $\mathrm{CH}$. We discuss our findings across these three examinations and propose future directions of research acknowledging methodological caveats.

\section{The mechanisms of action of GONB}

We successfully identified rCBF changes in $\mathrm{CH}$ patients following GONB administration. Firstly, we observed rCBF reductions in lobule VIII of left cerebellum in all patients following treatment as well as in comparison to healthy controls. The connection between cerebellum and other structures commonly linked to $\mathrm{CH}$ mechanisms, such as VTA and hypothalamus, has been previously demonstrated in healthy individuals and $\mathrm{CH}$ patients and decreases in cerebellar metabolism in $\mathrm{CH}$ patients during hypothalamic deep brain stimulation have also been described [30]. Comparably, vagus nerve stimulation, currently used for suppressing $\mathrm{CH}$ attacks $[31,32]$, can provoke changes in $\mathrm{rCBF}$ in the inferior 


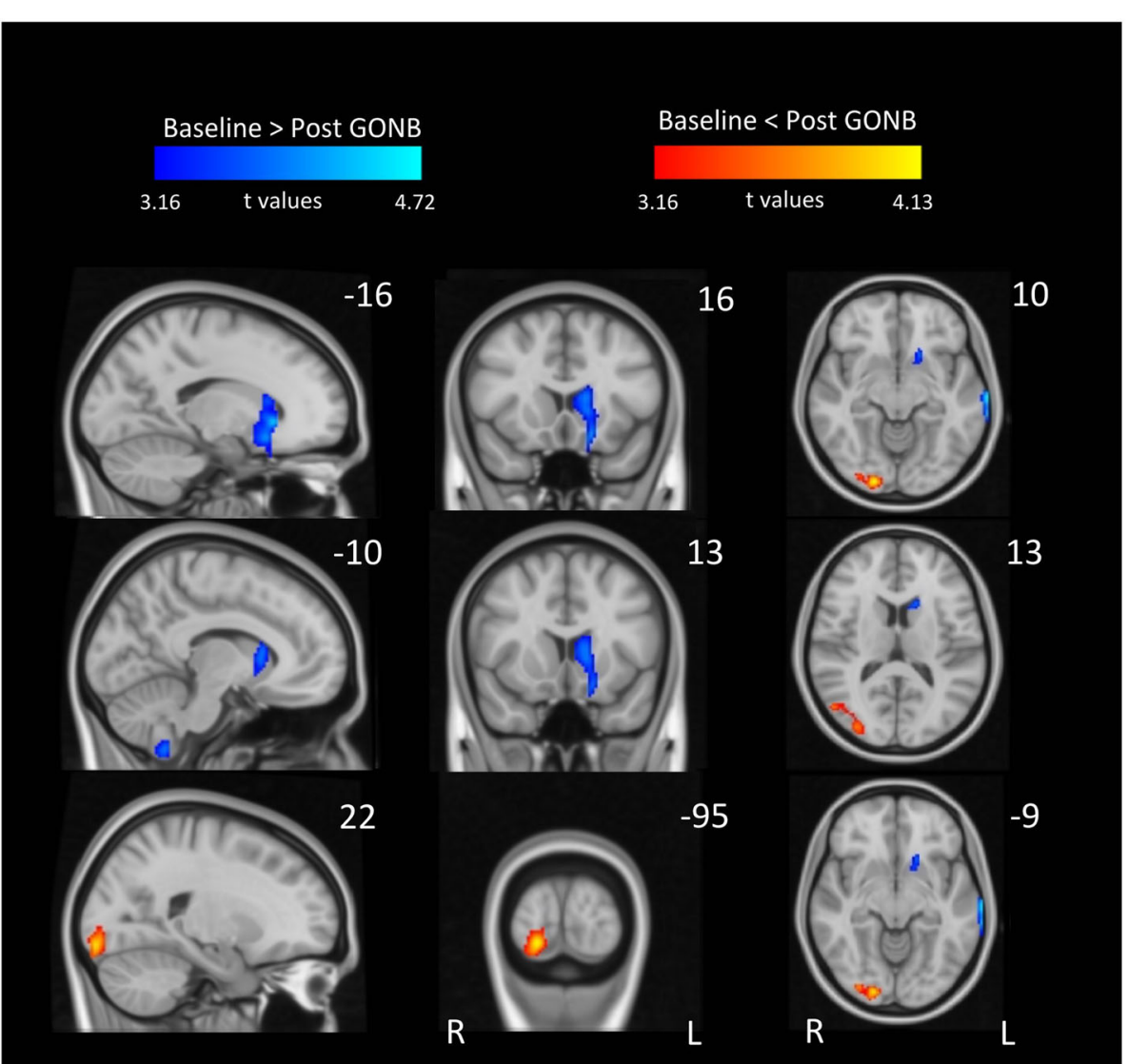

Fig. 2 Local decreases (blue) and increases (red) of rCBF across all CH patients following GONB. Brain areas in blue include medial temporal gyrus, cerebellum and substructures of basal ganglia including caudate and putamen. Cluster in red colour corresponds to secondary visual cortex. Data included four CBF maps per patient and session. All clusters are significant at $p<0.05$ (FWE corrected; initial height threshold set to 0.001). GONB = greater occipital nerve block; $R=$ right; $L=$ left

cerebellum in epileptic patients [33], and its mechanism of action has been linked to changes in the hypothalamic-pituitary-adrenal axis [34]. Taken together, our results support the theories of a modulatory function of the hypothalamic-cerebellar pathway in $\mathrm{CH}$. Despite not observing perfusion changes in these midbrain areas in our data, such changes did emerge when using a more liberal cluster-forming threshold, commonly used in previous MRI literature (Fig. 5).

We observed increases in $\mathrm{rCBF}$ in right secondary visual cortex after GONB across patients. In contrast, perfusion decreases in primary visual cortex after GONB, as well as in comparison to healthy controls have been previously reported [35]; the authors speculated that these differences could be due to the existence of visual aura in $\mathrm{CH}$ patients. However, since we scanned patients during asymptomatic headache-free periods, interpreting our results in relation to aura is challenging. Nevertheless, responders to GONB demonstrated greater $\mathrm{rCBF}$ both at baseline and following GONB, compared to non- responders, in the right lateral occipital complex (LOC). The LOC has been shown to modulate pain memory [36] and it has been suggested to be involved in the lateralisation of $\mathrm{CH}$ attacks [37]. In fact, evoked trigeminal pain in healthy individuals interrupts visual encoding in this group of areas [38]. Our results, in line with these findings, indicate that the pathophysiology of $\mathrm{CH}$ may well extend beyond areas commonly associated with pain experience [39], pointing towards the integration of pain structures and superior areas of the visual system in the pathogenesis of $\mathrm{CH}$.

Finally, following GONB we observed decreased perfusion across patients in the dorsal striatum. The striatum is well known for playing a major role in endogenous analgesia [40]. It is directly connected to the trigeminal nucleus caudalis, which is the primary target for GONB afferent inhibition [41] and its analgesic effect relies on activation and propagation of dopamine $\mathrm{D}_{2}$ receptors towards the trigeminal nerve via basal ganglia. Specifically in $\mathrm{CH}$, increased axial diffusivity in the caudate nucleus 
Table 3 Summary of peak coordinates across all contrasts

\begin{tabular}{|c|c|c|c|c|c|c|c|c|c|c|c|}
\hline \multirow[t]{2}{*}{ Contrast } & & \multirow[t]{2}{*}{ Cluster } & \multirow[t]{2}{*}{ Side } & \multicolumn{3}{|c|}{$\begin{array}{l}\text { Peak } \\
\text { coordinates } \\
\text { (MNI) }\end{array}$} & \multirow[t]{2}{*}{$\begin{array}{l}\text { Cluster } \\
\text { size }\end{array}$} & \multirow[t]{2}{*}{$\mathbf{t}$} & \multirow[t]{2}{*}{$P_{(\mathrm{FWE})}$} & \multirow[t]{2}{*}{$\begin{array}{l}\text { Cohen's } \\
\text { d }\end{array}$} & \multirow[t]{2}{*}{$\begin{array}{l}\text { Hedge's } \\
\text { g }\end{array}$} \\
\hline & & & & $\mathbf{x}$ & $y$ & $\mathbf{z}$ & & & & & \\
\hline \multirow[t]{4}{*}{ Across all $\mathrm{CH}$ patients } & $\begin{array}{l}\text { Pre }>\text { Post GONB } \\
\text { treatment }\end{array}$ & medial temporal gyrus & left & -70 & -20 & -14 & 347 & 4.72 & 0.017 & 0.39 & 0.39 \\
\hline & & $\begin{array}{l}\text { cerebellum (lobule ix } \\
\text { extending to lobule viii) }\end{array}$ & left & -6 & -54 & -60 & 351 & 4.48 & 0.016 & 0.37 & 0.37 \\
\hline & & $\begin{array}{l}\text { caudate extending to } \\
\text { putamen }\end{array}$ & left & -16 & 22 & -4 & 430 & 4.34 & 0.006 & 0.36 & 0.36 \\
\hline & $\begin{array}{l}\text { Pre }<\text { Post GONB } \\
\text { treatment }\end{array}$ & $\begin{array}{l}\text { secondary visual cortex } \\
\text { (ba18) }\end{array}$ & right & 20 & -92 & -12 & 618 & 4.13 & 0.001 & 0.35 & 0.34 \\
\hline \multirow{2}{*}{$\begin{array}{l}\text { Responders vs Non- } \\
\text { responders to GONB at } \\
\text { BASELINE }\end{array}$} & $\begin{array}{l}\text { Responders > } \\
\text { Non-responders }\end{array}$ & lateral occipital cortex & right & 38 & -74 & 20 & 333 & 5.32 & 0.008 & 1.29 & 1.28 \\
\hline & $\begin{array}{l}\text { Responders < } \\
\text { Non-responders }\end{array}$ & $\begin{array}{l}\text { posterior cingulate gyrus } \\
\text { extending to primary motor } \\
\text { cortex }\end{array}$ & right & 2 & -30 & 48 & 448 & 5.18 & 0.002 & 1.26 & 1.24 \\
\hline \multirow{4}{*}{$\begin{array}{l}\text { Responders vs Non- } \\
\text { responders to GONB AFTER } \\
\text { treatment }\end{array}$} & $\begin{array}{l}\text { Responders > } \\
\text { Non-responders }\end{array}$ & $\begin{array}{l}\text { superior lateral occipital } \\
\text { cortex }\end{array}$ & right & 38 & -74 & 20 & 1508 & 7.24 & $\begin{array}{l}< \\
0.001\end{array}$ & 1.76 & 1.74 \\
\hline & $\begin{array}{l}\text { Responders }< \\
\text { Non-responders }\end{array}$ & posterior cingulate cortex & right & -4 & -26 & 18 & 272 & 4.41 & 0.034 & 1.07 & 1.06 \\
\hline & & middle temporal gyrus & left & -68 & -46 & 2 & 312 & 5.03 & 0.019 & 1.22 & 1.21 \\
\hline & $\begin{array}{l}\text { Responders }>\text { Non- } \\
\text { responders (SVC) }\end{array}$ & medial prefrontal cortex & left & -12 & 52 & 14 & 115 & 5.56 & $0.015^{*}$ & 1.35 & 1.34 \\
\hline \multirow[t]{5}{*}{$\begin{array}{l}\mathrm{CH} \text { patients vs Healthy } \\
\text { controls }\end{array}$} & $\begin{array}{l}\text { CH Patients > } \\
\text { Health controls }\end{array}$ & cerebellum (lobule viii) & left & -14 & -62 & -42 & 219 & 4.82 & 0.025 & 2.15 & 2.08 \\
\hline & & hippocampus & left & -34 & -30 & -14 & 756 & 4.79 & $\begin{array}{l}< \\
0.001\end{array}$ & 2.13 & 2.06 \\
\hline & $\begin{array}{l}\text { CH Patients < } \\
\text { Health controls }\end{array}$ & orbitofrontal cortex & right & 22 & 56 & -6 & 1896 & 7.97 & $\begin{array}{l}< \\
0.001\end{array}$ & 3.55 & 3.43 \\
\hline & & rostral anterior insula & right & 52 & 18 & 2 & 218 & 6.77 & 0.025 & 3.02 & 2.92 \\
\hline & & middle temporal gyrus & right & 54 & -18 & -16 & 372 & 6.45 & 0.002 & 2.87 & 2.78 \\
\hline
\end{tabular}

CH cluster headache, GONB greater occipital nerve block, BA Broadman area, SVC small volume correction

in $\mathrm{CH}$ patients compared to controls has been reported [42] suggesting altered neural pain-related plasticity in these patients. Neuropeptide studies may shed further light into how these results relate to a $\mathrm{CBF}$ reduction in the striatum after GONB, as they may provide with a comprehensive description of the molecular processes taking place between the striatum and the trigeminovascular nociceptive pathways [43]. Admittedly, our results from perfusion before vs after GONB comparisons yield a modest effect size, as indicated by Hedge's $g$ figures that gravitate between 0.35 and 0.39 (Table 3), which may well be a direct consequence of our relatively low sample size; however, this first attempt to characterise mechanisms of GONB responses in an arguably rare clinical cohort like $\mathrm{CH}$ patients should facilitate future highly powered hypothesis-driven versions, including replications, of the present experimental design. It is important to stress that the CBF changes following GONB discussed above are unlikely to relate to the effects of corticosteroid intervention, not only because medication remained stable throughout the study and therefore any variability would be across patients and not withinparticipants, but also because only two patients included in the analyses were on prednisolone treatment, precluding a main effect of the drug to emerge at group level. One could also argue that the reduction of $\mathrm{CH}$ attacks following treatment across ECH patients could be due to the natural remission of their bout; this was accounted for in out design, and accordingly, we made sure both study visits were scheduled before their bout was expected to end, aiming always for the first half of their bouts. Nevertheless, and despite our best efforts, we cannot rule out that in some exceptional cases the bouts ended earlier than usual, a factor that always should be taken into account when determining responses to treatment in episodic $\mathrm{CH}$ patients. 
Baseline

Responders $>$ non-responders Responders $<$ non-responders

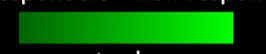

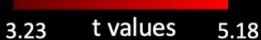
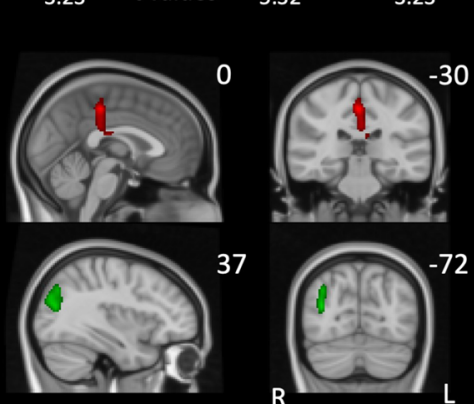

37

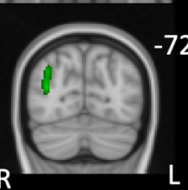

SVC
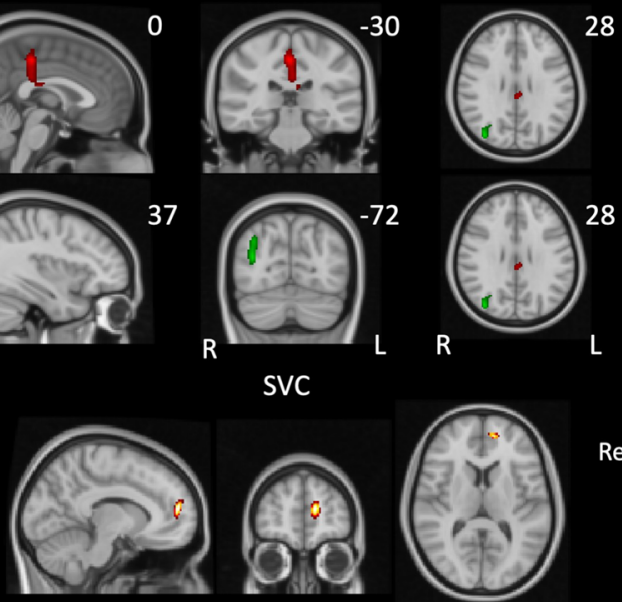

28

$\mathbf{R}$

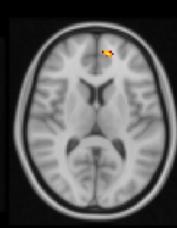

L

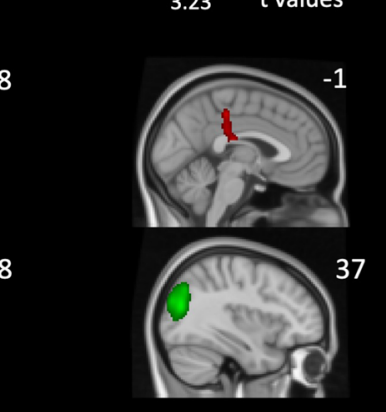

Responders > non-responders Responders $<$ non-responders
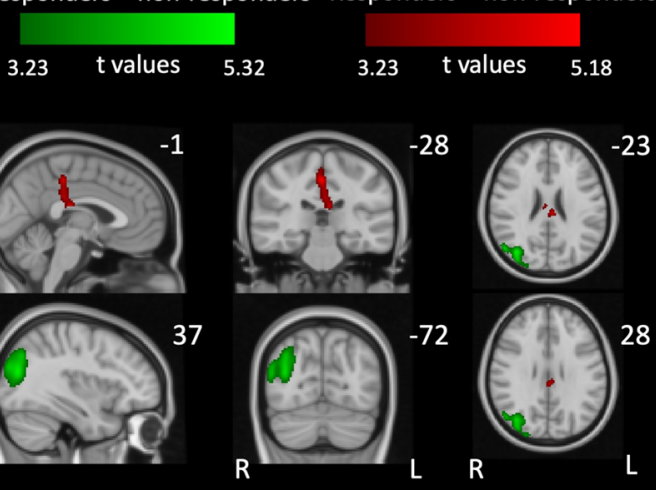

Baseline

Responders $>$ non-responders (SVC)

3.23

$\mathrm{t}$ values

Fig. 3 Differences in rCBF between the responders and the non-responders groups of $\mathrm{CH}$ patients at baseline (left panel) and following GONB (right panel). Increased local perfusion in responders (green) is observed in the LOC both prior and after GONB, being extended in the post GONB session. Increased local perfusion in non-responders comprised the PCC and the PMC in both sessions. Lower panel shows increased activation in mPFC for the responders group after performing small volume correction (SVC). Data included four CBF maps per patient and session. All clusters are significant at $p<0.05$ (FWE corrected; initial height threshold set to 0.001). GONB = greater occipital nerve block; $R=$ right; $L=$ left

\section{Prediction of treatment response}

It remains critical to identify brain characteristics at baseline that may indicate the likely efficacy of GONB in suppressing $\mathrm{CH}$ attacks in individual patients. We investigated this question via examination of differences in rCBF at prior to GONB between responders and nonresponders.

In line with our a priori hypothesis, we observed greater $\mathrm{CBF}$ in the medial PFC in responders at baseline compared to non-responders. This result was not driven by differences in depression, anxiety or quality of life measures between the two groups. The role of the medial PFC in anticipation of placebo analgesia has already been described in healthy volunteers, ${ }^{46}$ and the extent of central hyperalgesia is negatively correlated with activity in this area [44]. Additionally, placebo effects have been linked to differential treatment responses in $\mathrm{CH}$ [45], however, imaging evidence on placebo effects in $\mathrm{CH}$ has yet to be reported. A limitation of our study is the absence of placebo control arm, but it is arguable that the medial PFC is indeed involved in mediating response in the active treatment arm. The implication that the medial PFC may be involved in mediating response to both active and placebo treatment needs further investigation.
We identified reduced local CBF in the posterior cingulate cortex (PCC) that extended to the primary motor cortex in responders in comparison to non-responders, that were unaltered by GONB. The PCC is involved in integration of memories, motivational-affective components [46], and ruminating thoughts during pain [21]. Moreover, enhanced perfusion compared to healthy individuals in this area has been suggested to be an increased orientation of attention towards pain in osteoarthritis patients [47]. Together, our results suggest that differences in the PCC might relate to patients' psychological states in relation to $\mathrm{CH}$. We speculate that more negative beliefs and ruminating thoughts towards the condition and the future are likely to relate treatment efficacy.

We identified decreases in rCBF in left medial temporal gyrus (MTG) across patients following treatment, as well as lower rCBF in the responders group compared to non-responders at baseline. Increased perfusion in MTG in $\mathrm{CH}$ patients when comparing scans in bout vs out of bout has been reported [13]; further, reduced FC between hypothalamus and MTG [48] and reduced GM in MTG [29] have been shown in $\mathrm{CH}$ patients compared to healthy individuals. Importantly, MTG activation 


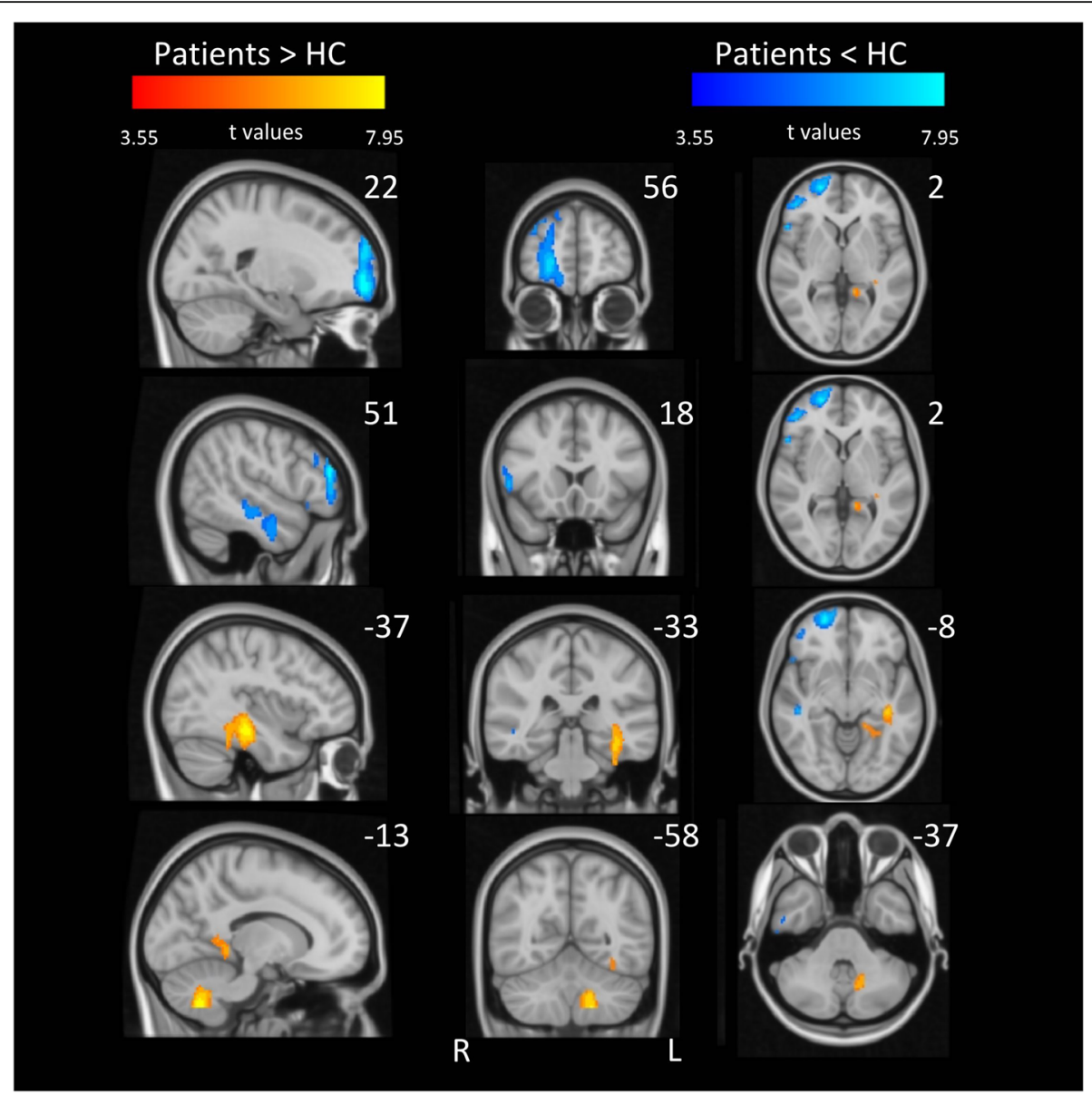

Fig. 4 Local increases (red) and decreases (blue) in CBF across all CH prior to GONB in comparison to healthy controls. Brain areas in red include cerebellum and hippocampus. Clusters in blue colour corresponds to OFC, primary auditory cortex, insula and MTG. For these contrasts, data included one CBF map per patient and healthy individual. All clusters are significant at $p<0.05$ (FWE corrected; initial height threshold set to 0.001 )

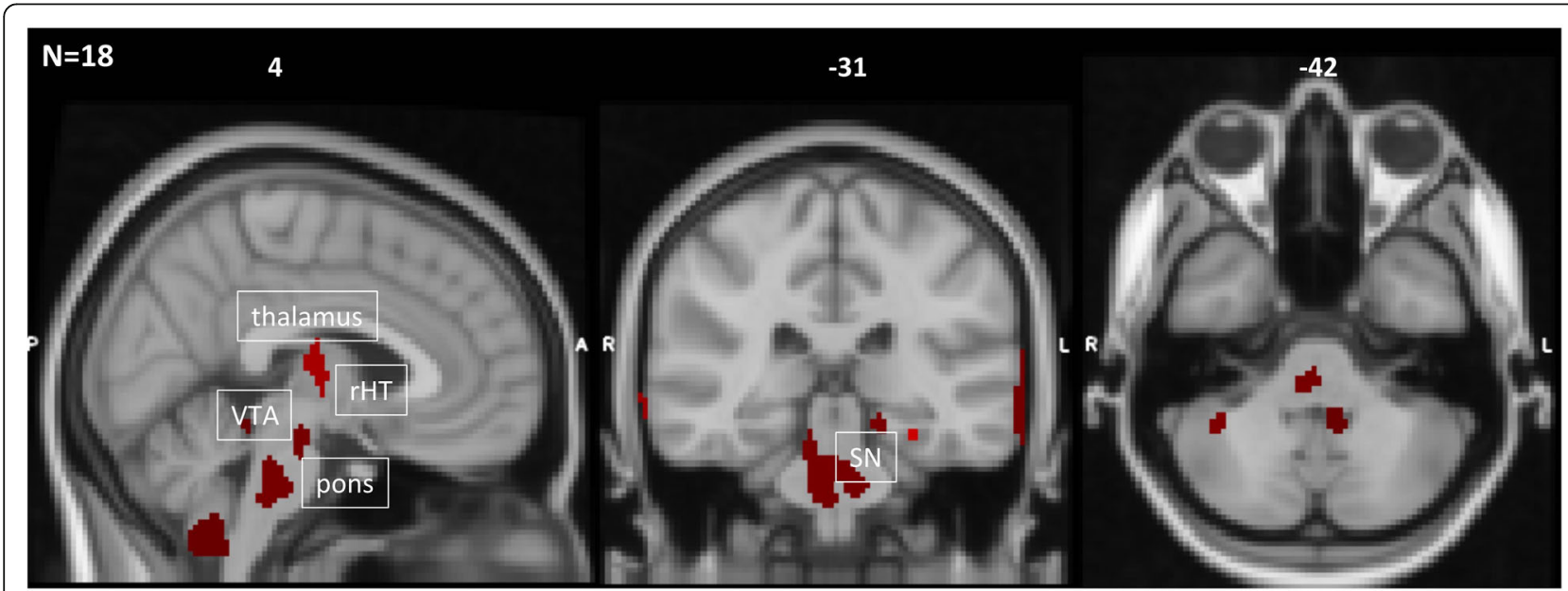

Fig. 5 Exploratory results from local decreases in CBF across all CH patients following GONB at more relaxed initial cluster-forming height threshold. Data included four CBF maps per patient and session. All clusters are significant at $p<0.005$ (uncorr). rHT = right hypothalamus; VTA $=$ ventral tegmental area; $\mathrm{SN}=$ substantia nigra. Coordinates are represented in MNI space 
seems to be involved in inhibitory function during conditioned pain modulation [49] and it has been linked to pain recognition in others [50]. Since the MTG is classically linked to recognition and retrieval if concepts, our results, together with existing evidence, hint that impaired meaning attribution of pain-related information in the MTG might play a role in the pathophysiology of $\mathrm{CH}$.

\section{$\mathrm{CH}$ patients vs healthy controls}

We observed greater $\mathrm{rCBF}$ in the orbitofrontal cortex (OFC) in healthy controls in comparison to patients. It has been suggested that reduced GMV in OFC contributes to poorer top-down inhibitory control of pain signals in chronic pain, including $\mathrm{CH}$ [14]. These findings suggest that perturbations in OFC may impair chronic pain patients' capacity to manage afferent nociceptive signals.

We also identified greater $\mathrm{rCBF}$ in the dorsal hippocampus in $\mathrm{CH}$ patients compared to healthy controls. GMV reductions in $\mathrm{CH}$ patients compared to healthy controls have been reported [16], that develop and change with time and disease stage, suggesting that the hippocampus could be involved in pain memory, and its activation is related to pain expectancy and harm avoidance [51]. The hippocampus is one of the main mediators of anxiety in pain processing [52]. It is plausible that anxiety-related personality traits, as indexed by our borderline HADS results, are playing a role in the emergence $\mathrm{CH}$ symptoms by priming memories of previous headache attacks and facilitating pain states. Furthermore, we observed decreased $\mathrm{rCBF}$ in the rostral anterior insula in the $\mathrm{CH}$ patients group compared to healthy controls at baseline. Decreases in GMV in the anterior insula in $\mathrm{CH}$ patients out of bout versus healthy controls have been previously reported [16]. Some authors argue that the rostral anterior insula is a specific locus for clinical pain, regardless of the pain condition [53], suggesting a distinct pathway impairment in these patients. Qiu et al. [54] found decreased FC between the hypothalamus and the salience network, of which the anterior insula is a key component, in pain-free $\mathrm{CH}$ patients in bout compared to healthy controls. Importantly, the hypothalamus is heavily involved in stress regulation through the hypothalamus-pituitary-adrenal axis [55]. Our results stand in line with these claims, suggesting that a combination of higher stress response facilitation towards pain, alongside with impaired stress-related affective response control may be elements that ultimately, contribute to the chronification of headache attacks.

Despite the fact that our results involving differences between responders and non-responders, as well as between $\mathrm{CH}$ patients and healthy controls are indeed derived from a low number of participants, the corrected effect size estimations for each of the contrasts are largely above the accepted cut off for an effect considered as 'large' (e.g. Hedge's g > 0.80), serving as a first indicator of the robustness of the data discussed above. Nonetheless, forthcoming studies including a larger number of patients and healthy controls may replicate these findings and shed further light into perfusion patters that may reliably act as predictors of GONB response in $\mathrm{CH}$ patients.

\section{Conclusions}

In summary, our results indicate that the pathophysiology of $\mathrm{CH}$ includes, but is not limited to, brain areas typically linked to pain perception; while changes in brain perfusion after GONB point out as possible main targets areas innervated by the trigeminal nerve (i.e. cerebellum, striatum, visual cortex), we propose that there is a heavy psychological component that might be driving treatment responses through poor anxiety and stress response regulation, attentional bias towards pain, and ruminating thoughts; our results point to differences in areas previously associated with these psychological states at baseline. Future research may elucidate whether response to GONB may be improved by combining it with therapies focused on controlling negative thoughts towards pain promoting cognitive flexibility. Likewise, further investigation of GONB responses including placebo-controlled designs might disentangle differential responses to treatment. Overall, our findings provide further characterisation of underlying brain mechanisms in $\mathrm{CH}$ that extend beyond the traditional midbrain hubs widely discussed in the literature.

\section{Abbreviations}

CH: Cluster Headache; GONB: Greater Occipital Nerve Block; rCBF: Regional Cerebral Blood Flow; MRI: Magnetic Resonance Imaging; BOLD: Blood Oxygenation Level Dependant; ASL: Arterial Spin Labelling; pCASL: Pseudo Continuous Arterial Spin Labelling; GMV: Grey Matter Volume; PFC: Prefrontal Cortex; HADS: Hospital Anxiety and Depression Scale; SF-36: 36-item Short Form Survey; FSE: Fast Spin Echo; FSL: FMRIB Software Library; MNI: Montreal Neurological Institute; SPM: Statistical Parametric Mapping; FWE: Family Wise Error; ANOVA: Analysis of Variance; CSF: Cerebrospinal Fluid; SVC: Small Volume Correction; ROI: Region of Interest; VTA: Ventral Tegmental Area; LOC: Lateral Occipital Cortex; PCC: Posterior Cingulate Cortex; MTG: Medial Temporal Gyrus; OFC: Orbitofrontal Cortex

\section{Acknowledgements}

SM, MAH, OOD and SCW are supported by the NIHR Biomedical Research Centre for Mental Health at the South London and Maudsley NHS Trust. SM, MAH, TFR and SCW are also supported by a Medical Research Council EMCG grant (MR/N026969/1). We would like to thank The Government of Brunei for funding the present study. We would like to thank Dr. Jade Jackson, Dr. Francesca Puledda and Dr. Philip Holland for their valuable comments and suggestions.

\section{Authors' contributions}

SM contributed to drafting the manuscript, preparing figures, data sourcing form healthy controls, data analysis completion and data interpretation. NAB contributed to the study design, data acquisition and revision of the manuscript for intellectual content. OOD made substantial contribution to 
data analysis advice and revision of manuscript for intellectual content. SaM contributed to the study design and data acquisition. EM contributed with substantial data analysis advice and revision of manuscript for intellectual content. TR contributed to the study conception and experimental design. SCRW contributed to the study conception and design, and revision of the manuscript for intellectual content. MM contributed to the study conception and design, data acquisition and substantial revision of manuscript for intellectual content. MAH contributed to the study conception and design, data analysis advice and revision and revision of manuscript for intellectual content. The author(s) read and approved the final manuscript.

\section{Funding}

The present study was funded by an In-Service Training Scholarship Scheme from the Government of His Majesty The Sultan and Yang Di-Pertuan of Brunei Darussalam. SM, MAH, OOD and SCW are supported by the NIHR Biomedical Research Centre for Mental Health at the South London and Maudsley NHS Trust. SM, MAH, TFR and SCW are also supported by a Medical Research Council EMCG grant (MR/N026969/1).

\section{Availability of data and materials}

All data were acquired at the Centre for Neuroimaging Sciences at King's College London. Composite groupwise statistical maps derived study analyses are available from the corresponding author SM on reasonable request.

\section{Declarations}

\section{Ethics approval and consent to participate}

The study was approved by the National Research Ethics Service [LondonQueen Square Research Ethics Committee (reference: 12/LO/0419)]. All participants provided written informed consent prior to participation according to the Declaration of Helsinki.

\section{Consent for publication}

Not applicable.

\section{Competing interests}

Within the past 2 years (and during the course of the study under consideration if the study exceeded 2 years), author MM received personal compensation for serving on a scientific advisory board at Abbott, Allergan Medtronic, TEVA, Novartis and Eli Lilly.

The other authors declare that they have no competing interests.

\section{Author details}

'Department of Neuroimaging, King's College London, Institute of Psychiatry, Psychology and Neuroscience, Box 89, De Crespigny Park, London SE5 8AF, UK. 'Wolfson Centre for Age-Related Diseases, King's College London, London, UK. ${ }^{3}$ Department of Oral Surgery, King's College London, London, UK. ${ }^{4}$ Headache and Facial Pain Group, UCL Queen Square Institute of Neurology, Queen Square, London, UK.

\section{Received: 19 May 2021 Accepted: 30 July 2021}

\section{Published online: 12 August 2021}

\section{References}

1. Kingston WS, Dodick DW (2018) Treatment of cluster headache. Ann Indian Acad Neurol 21(Suppl 1):S9-S15

2. Lambru G et al (2014) Greater occipital nerve blocks in chronic cluster headache: a prospective open-label study. Eur J Neurol 21(2):338-343

3. Busch $V$ et al (2007) Occipital nerve blockade in chronic cluster headache patients and functional connectivity between trigeminal and occipital nerves. Cephalalgia 27(11):1206-1214

4. Norris J, Hachinski V, Cooper P (1976) Cerebral blood flow changes in cluster headache. Acta Neurol Scand 54(4):371-374

5. May A et al (1998) Hypothalamic activation in cluster headache attacks. Lancet 352(9124):275-278

6. Teepker $\mathrm{M}$ et al (2012) Diffusion tensor imaging in episodic cluster headache. Headache J Head Face Pain 52(2):274-282

7. Wang $R$ et al (2014) Cognitive processing of cluster headache patients: evidence from event-related potentials. J Headache Pain 15(1):66-66
8. Rocca MA et al (2010) Central nervous system dysregulation extends beyond the pain-matrix network in cluster headache. Cephalalgia 30(11): 1383-1391

9. Morelli N et al (2013) Brainstem activation in cluster headache: an adaptive behavioural response? Cephalalgia 33(6):416-420

10. Qiu E et al (2013) Abnormal brain functional connectivity of the hypothalamus in cluster headaches. PLoS One 8(2):e57896

11. Matharu MS, Zrinzo L (2010) Deep brain stimulation in cluster headache: hypothalamus or midbrain Tegmentum? Curr Pain Headache Rep 14(2):151159

12. Loggia ML et al (2019) Imaging clinically relevant pain states using arterial spin labeling. Pain reports 4(4):e750

13. Sprenger $T$ et al (2007) Altered metabolism in frontal brain circuits in cluster headache. Cephalalgia 27(9):1033-1042

14. Yang F-C, et al (2013) Altered gray matter volume in the frontal pain modulation network in patients with cluster headache. PAIN ${ }^{\circledR}$ 154(6): 801-807

15. Absinta M et al (2012) Selective decreased grey matter volume of the painmatrix network in cluster headache. Cephalalgia 32(2):109-115

16. Naegel $\mathrm{S}$ et al (2014) Cortical plasticity in episodic and chronic cluster headache. Neurolmage: Clinical 6:415-423

17. Dusi $\mathrm{N}$ et al (2015) Brain structural effects of antidepressant treatment in major depression. Curr Neuropharmacol 13(4):458-465

18. Kujawa A et al (2016) Prefrontal reactivity to social signals of threat as a predictor of treatment response in anxious youth. Neuropsychopharmacol 41(8):1983-1990

19. lacovides S, Avidon I, Baker F (2015) Does pain vary across the menstrual cycle? A review. Eur J Pain 19(10):1389-1405

20. The International Classification of Headache Disorders (2013) 3rd edition (beta version). Cephalalgia 33(9):629-808

21. Kucyi A, Davis KD (2015) The dynamic pain connectome. Trends Neurosci 38(2):86-95

22. Xu F et al (2012) Effect of hypoxia and Hyperoxia on cerebral blood flow, blood oxygenation, and oxidative metabolism. J Cereb Blood Flow Metab 32(10):1909-1918

23. Wise RG et al (2013) Measurement of OEF and absolute CMRO2: MRI-based methods using interleaved and combined hypercapnia and hyperoxia. Neuroimage 83:135-147

24. Bjelland I et al (2002) The validity of the hospital anxiety and depression scale: an updated literature review. J Psychosom Res 52(2):69-77

25. Autret A et al (2010) Psychopathology and quality of life burden in chronic daily headache: influence of migraine symptoms. J Headache Pain 11(3): 247-253

26. Baguley T (2012) Serious stats: A guide to advanced statistics for the behavioral sciences. Macmillan International Higher Education

27. Pustejovsky JE (2014) Converting from d to $r$ to $z$ when the design uses extreme groups, dichotomization, or experimental control. Psychol Methods 19(1):92-112

28. Hedges L, Olkin I (2014) Statistical methods for meta-analysis. Academic press

29. Absinta M et al (2011) Selective grey matter atrophy in the pain-matrix network in cluster headache: O237. J Neurol 258

30. May A et al (2006) Hypothalamic deep brain stimulation in positron emission tomography. J Neurosci 26(13):3589-3593

31. Silberstein SD et al (2016) Non-invasive Vagus nerve stimulation for the ACute treatment of cluster headache: findings from the randomized, double-blind, sham-controlled ACT1 study. Headache J Head Face Pain 56(8):1317-1332

32. Gaul C et al (2015) Non-invasive vagus nerve stimulation for PREVention and Acute treatment of chronic cluster headache (PREVA): a randomised controlled study. Cephalalgia 36(6):534-546

33. Ko D et al (1996) Vagus nerve stimulation activates central nervous system structures in epileptic patients during PET H215O blood flow imaging. Neurosurgery 39(2):426-431

34. O'Keane $\mathrm{V}$ et al (2005) Changes in hypothalamic-pituitary-adrenal Axis measures after Vagus nerve stimulation therapy in chronic depression. Biol Psychiatry 58(12):963-968

35. Magis D et al (2011) Central modulation in cluster headache patients treated with occipital nerve stimulation: an FDG-PET study. BMC Neurol 11(1):25

36. Bingel $U$ et al (2007) fMRI reveals how pain modulates visual object processing in the ventral visual stream. Neuron 55(1):157-167 
37. Boiardi A et al (1986) Visual evoked potentials in cluster headache: central structures involvement. Headache J Head Face Pain 26(2):70-73

38. Schmidt $\mathrm{K}$ et al (2016) The differential effect of trigeminal vs. peripheral pain stimulation on visual processing and memory encoding is influenced by pain-related fear. Neurolmage 134:386-395

39. Wager TD et al (2013) An fMRl-based neurologic signature of physical pain. N Engl J Med 368(15):1388-1397

40. Barceló AC, Filippini B, Pazo JH (2012) The striatum and pain modulation. Cell Mol Neurobiol 32(1):1-12

41. Ashkenazi A, Levin M (2007) Greater occipital nerve block for migraine and other headaches: is it useful? Curr Pain Headache Rep 11(3):231-235

42. Király A et al (2017) Macro- and microstructural alterations of the subcortical structures in episodic cluster headache. Cephalalgia 38(4):662-673

43. Buture A et al (2019) Update on the pathophysiology of cluster headache: imaging and neuropeptide studies. J Pain Res 12:269-281

44. Seifert $F$ et al (2009) Medial prefrontal cortex activity is predictive for hyperalgesia and pharmacological antihyperalgesia. J Neurosci 29(19):61676175

45. Mitsikostas DD, Mantonakis LI, Chalarakis NG (2011) Nocebo is the enemy, not placebo. A meta-analysis of reported side effects after placebo treatment in headaches. Cephalalgia 31(5):550-561

46. Bromm B (2004) The involvement of the posterior cingulate gyrus in phasic pain processing of humans. Neurosci Lett 361(1):245-249

47. Howard MA et al (2012) Alterations in resting-state regional cerebral blood flow demonstrate ongoing pain in osteoarthritis: an arterial spin-labeled magnetic resonance imaging study. Arthritis Rheumatism 64(12):3936-3946

48. Yang, F.-C., et al., Altered hypothalamic functional connectivity in cluster headache: a longitudinal resting-state functional MRI study. J Neurol Neurosurg Psychiatry. 2015: 86(4): 437

49. Moont, R., et al., Temporal changes in cortical activation during conditioned pain modulation (CPM), a LORETA study. PAIN ${ }^{\oplus}$. 2011;152(7): 1469-1477

50. Dozolme D et al (2018) The neuroelectric dynamics of the emotional anticipation of other people's pain. PLoS One 13(8):e0200535-e0200535

51. Ziv M et al (2010) Individual sensitivity to pain expectancy is related to differential activation of the hippocampus and amygdala. Hum Brain Mapp 31(2):326-338

52. Ploghaus A et al (2001) Exacerbation of pain by anxiety is associated with activity in a hippocampal network. J Neurosci 21(24):9896-9903

53. Schweinhardt $P$ et al (2006) An fMRI study of cerebral processing of brushevoked allodynia in neuropathic pain patients. Neurolmage 32(1):256-265

54. Qiu E et al (2015) Abnormal coactivation of the hypothalamus and salience network in patients with cluster headache. Neurology 84(14):1402

55. Blackburn-Munro G, Blackburn-Munro RE (2001) Chronic pain, chronic stress and depression: coincidence or consequence? J Neuroendocrinol 13(12): 1009-1023

\section{Publisher's Note}

Springer Nature remains neutral with regard to jurisdictional claims in published maps and institutional affiliations.

Ready to submit your research? Choose BMC and benefit from:

- fast, convenient online submission

- thorough peer review by experienced researchers in your field

- rapid publication on acceptance

- support for research data, including large and complex data types

- gold Open Access which fosters wider collaboration and increased citations

- maximum visibility for your research: over $100 \mathrm{M}$ website views per year

At $\mathrm{BMC}$, research is always in progress.

Learn more biomedcentral.com/submissions 